\title{
Correction: Deacetylation of HSPA5 by HDAC6 leads to GP78- mediated HSPA5 ubiquitination at K447 and suppresses metastasis of breast cancer
}

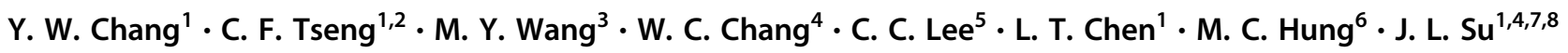 \\ Published online: 19 September 2019 \\ (c) Macmillan Publishers Limited 2019
}

\section{Correction to: Oncogene}

https://doi.org/10.1038/onc.2015.214

Since the publication of this article, the authors became aware of a number of errors which were missed at proof stage:

In Fig. 3d MCF7 should be replaced with MDA-MB231.

In Supplementary Fig. 2b MDA-MB-231 should be replaced with MCF7.

In Figs. 1b, 1e, 3c, and 4e and Supplementary Fig. 1MCF7 and MCF7/13 should be replaced with MDA-MB231 and MDA-MB-231/13, respectively.

The original article can be found online at https://doi.org/10.1038/onc. 2015.214.

$\triangle$ J. L. Su

hansoneblack@gmail.com

1 National Institute of Cancer Research, National Health Research Institutes, Zhunan, Taiwan

2 Graduate Program of Biotechnology in Medicine College of Life Science, National Tsing Hua University, Hsinchu, Taiwan

3 Department of Surgery, National Taiwan University Hospital, Taipei, Taiwan

4 Graduate Institute of Cancer Biology, China Medical University, Taichung, Taiwan

5 Core Facilities for Protein Structural Analysis, Academia Sinica, Taipei, Taiwan

6 Department of Molecular and Cellular Oncology, The University of Texas MD Anderson Cancer Center, Houston, TX, USA

7 Center for Molecular Medicine, China Medical University Hospital, Taichung, Taiwan

8 Department of Biotechnology, Asia University, Taichung, Taiwan
In Fig. 1b the image for MCF7 (previously labelled MDA-MB-231) was incorrect and has been replaced.

In Supplementary Fig. 1 the graph for MCF7 (previously labelled MDA-MB-231) was incorrect and has been replaced.

In Figs. 1d and 4a, the western blot images for Tubulin (Fig. 1a) and HA (Fig. 4a) were not correct and have been replaced.

In Fig. 3a, the western blot image was not correct. The authors mistakenly used the MDA-MB-231, HBLIOO and HS578T image instead of the MCF7/13, HBLIOO/13 and MDA-MB-231/13 image. The image has been replaced.

All corrected figures are included below. The results and conclusions of this article are not affected by these modifications. The authors apologise to readers for any inconvenience caused. 
b

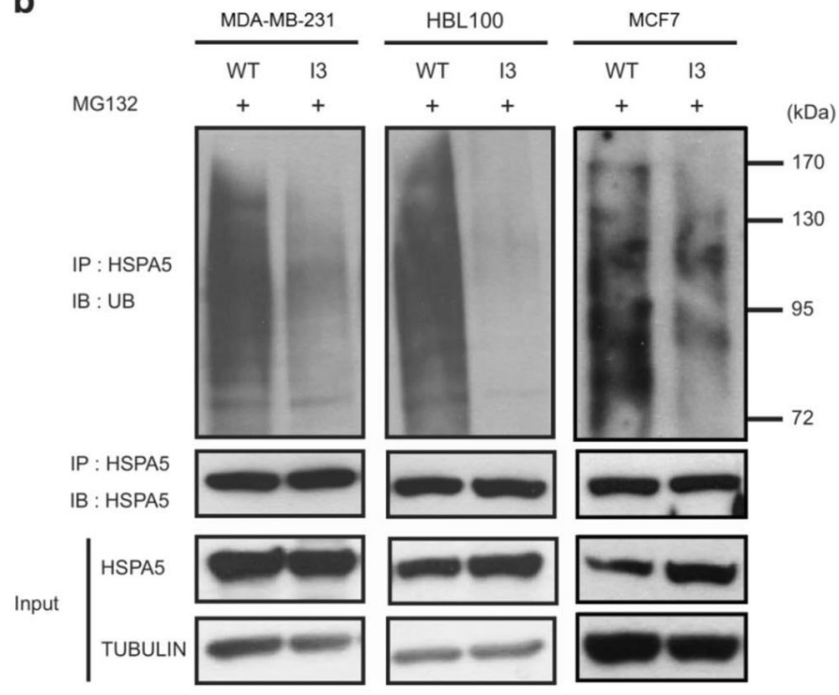

e

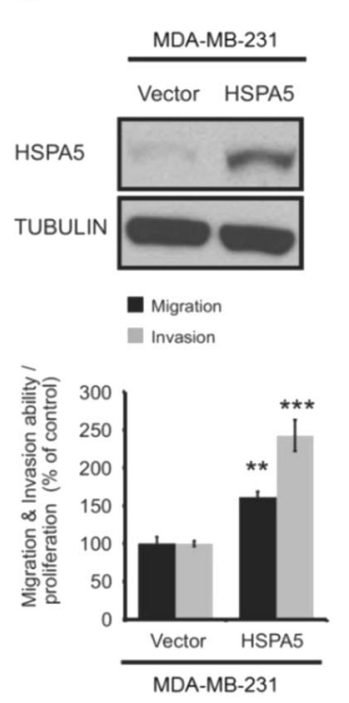

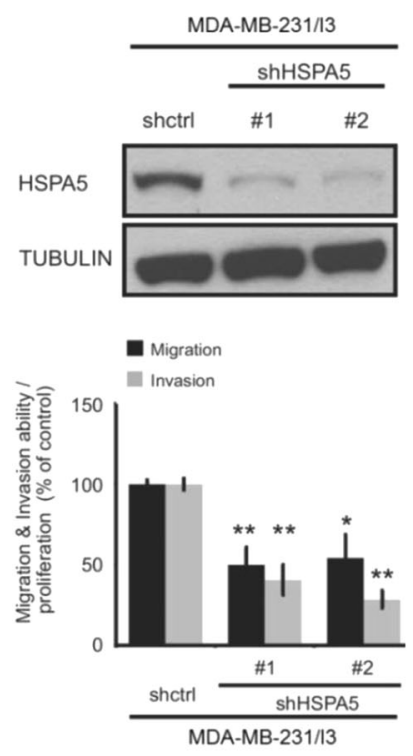

d

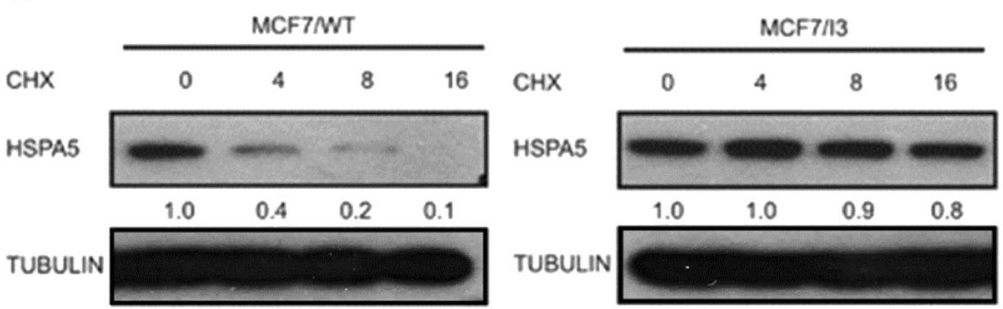

Fig. 1 Ubiquitin-proteasomal degradation of HSPA5 suppressed cell mobility. (a, top) HSPA5 expression in MCF7, HBL100 and MDAMB-231 cells was analyzed by western blot analysis. Tubulin was used as the internal protein loading control. (a, bottom) The migration and invasion abilities were measured in vitro with transwell assays, as described in the Materials and methods. Bars indicate means \pm s.d. of three independent experiments performed in triplicate. $* * P=0.01$; $* * * P=0.001$ compared with WT cells. b The WT and I3 cells of MCF7, HBL100 and MDA-MB-231 were treated with MG132 for $12 \mathrm{~h}$ and immunoprecipitated (IP) HSPA5 to detect ubiquitination of HSPA5. c MCF7/WT and MCF7/I3 cells were treated with or without proteasome inhibitor MG132 $(5 \mu \mathrm{M})$ for $12 \mathrm{~h}$, and HSPA5 protein expression was detected by western blot analysis. d MCF7/WT and MCF7/I3 cells were treated with $20 \mu \mathrm{g} / \mathrm{ml}$ cycloheximide (CHX) for indicated time points, and then detect HSPA5 protein expression by western blot analysis. e Western blot analysis of HSPA5 expression and the measurement of the migration and invasion abilities were measured by transwell assays in HSPA5-overexpressing MCF7 cells (left) and knockdown of HSPA5 in MCF7/I3 cells (right). Data are shown as mean \pm s.d. of three independent experiments performed in triplicate. $* P=0.05 ; * * P=0.01 ; * * * P=0.001$ 


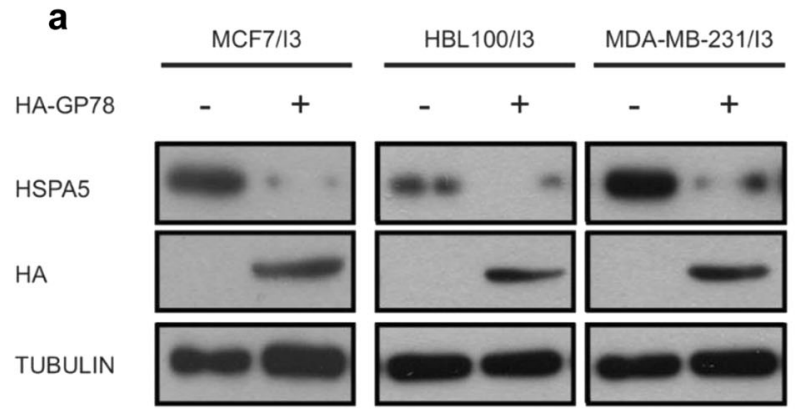

d

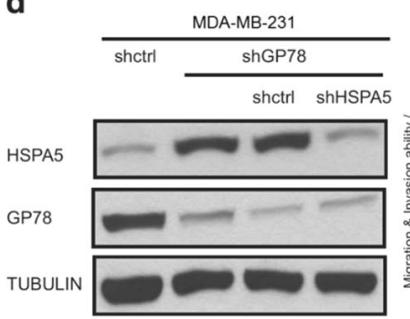

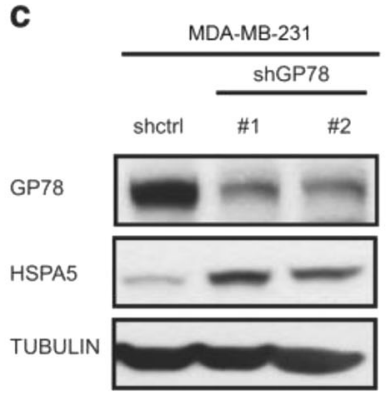

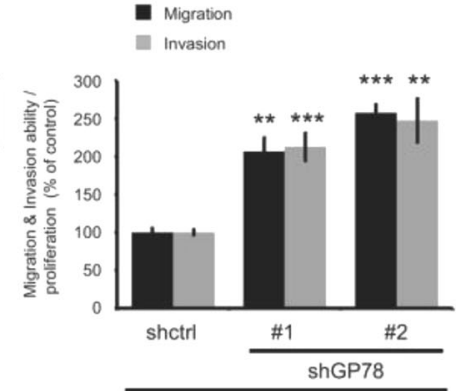

MDA-MB-231

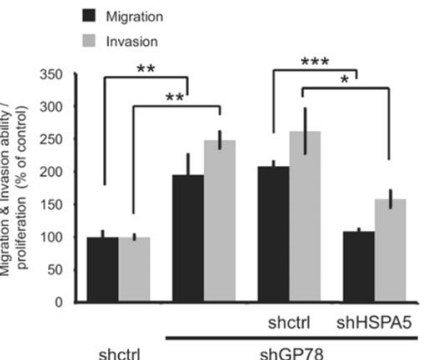

MDA-MB-231

Fig. 3 GP78 regulates cell migration/invasion through HSPA5 ubiquitination at K447. a MCF7, HBL100 and MDA-MB-231 cells were transfected with HA-tagged GP78 expression vector, and the expression of HA-GP78 and HSPA5 was confirmed by western blot analysis. b Determination of the protein stability of HSPA5 in MCF7/I3, MCF7/ WT, MCF7/WT/shctrl and MCF7/WT/shGP78 cells. Cells were treated with $20 \mu \mathrm{g} / \mathrm{ml}$ cycloheximide (CHX) for the indicated time, followed by western blot analysis. This was quantified by ImageJ system (Bethesda, MD, USA) and normalized to $0 \mathrm{~h}$ control. $* * P=0.01$; $* * * P=0.001$. Data are shown mean \pm s.d. of three independent experiments performed in triplicate. c MCF7 cells were transfected with shGP78 and shcontrol (shctrl) and submitted for western blot analysis (left) of HSPA5 expression and the measurement of the migration and invasion abilities by transwell assays (right). Data are shown as mean \pm s.d. of three independent experiments performed in triplicate. $* * P=0.01 ; * * * P=0.001$. d MCF7/shctrl and MCF7/ shGP78 cells were transfected with shHSPA5 and shctrl and submitted

for western blot analysis (left) of HSPA5 expression and the measurement of the migration and invasion abilities by transwell assays (right). Data are shown as mean \pm s.d. of three independent experiments performed in triplicate. $* P=0.05 ; * * P=0.01 ; * * * P=0.001$. e Representative bioluminescent images and quantification of lung colonization in mice injected with MDA-MB-231/shctrl, MDA-MB231/shGP78, MDA-MB-231/shGP78/shctrl and MDA-MB-231/ shGP78/shHSPA5 cells (six mice per group). Lung colonization was bioluminescently imaged after 8 weeks of injection. Data are shown as mean \pm s.e.m. of a minimum of six animals $(* * P=0.01)$. $\mathbf{f}$ HeLa cells were transfected with HSPA5-MYC/WT or HSPA5-MYC/K447A alone and in combination with HA-GP78 or vector control, and then the expression of HA-GP78 and HSPA5-MYC was confirmed by western blot analysis. $\mathrm{g}$ HeLa cellswere transfected with the indicated constructs and treated with MG132 for $12 \mathrm{~h}$. Total cell lysates were harvested and ubiquitination of HSPA5 was analyzed by immunoprecipation and western blot analysis 
a

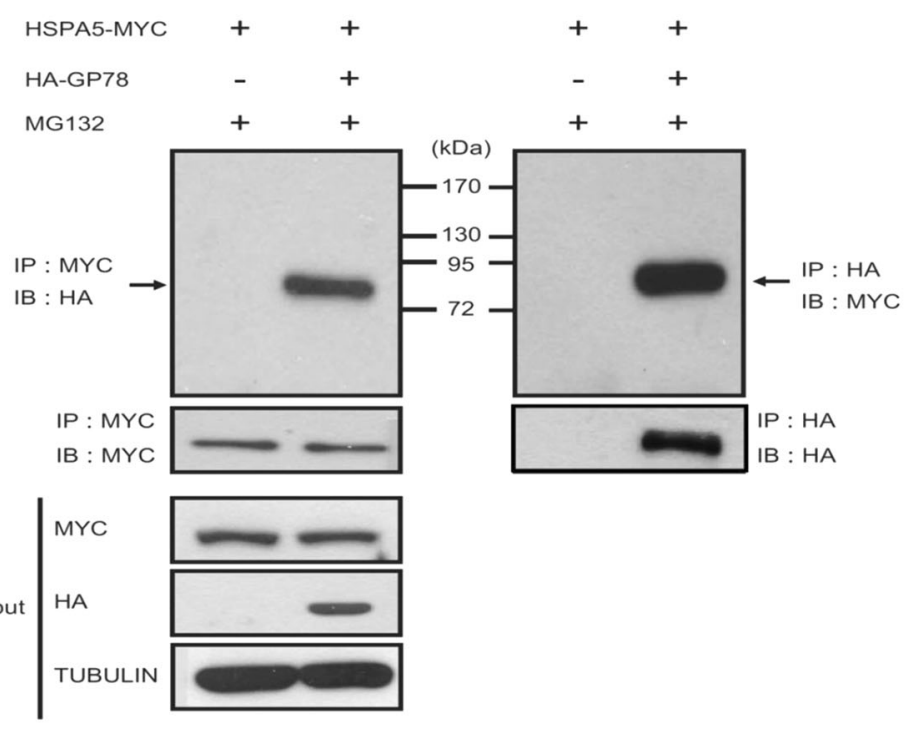

e

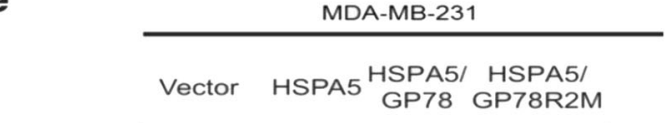

GP78

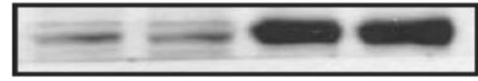

HSPA5

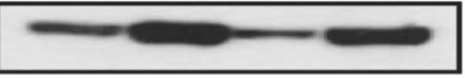

TUBULIN

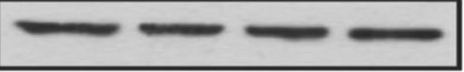

- Migration

Invasion

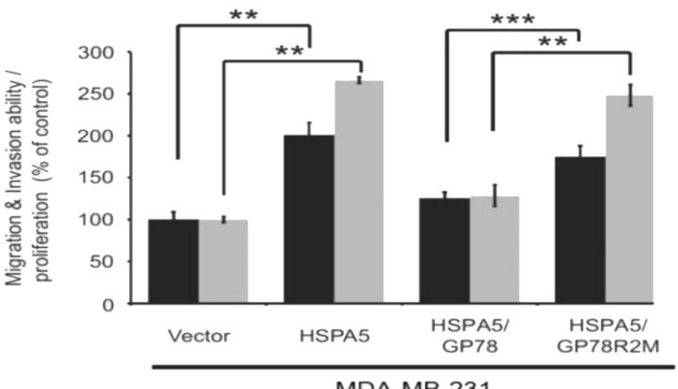

MDA-MB-231
Fig. 4 GP78 interacts with the C-terminal region of HSPA5 and decreases HSPA5 expression and HSPA5-mediated cell migration/ invasion. a HeLa cells were transfected with HSPA5-MYC alone or in combination with HA-GP78. Total cell lysates were prepared for IP and western blot analysis with the indicated antibodies. b Schematic representation of N-terminal HSPA5 (N-ter-MYC), C-terminal HSPA5 (C-ter-MYC) and full-length HSPA5 (HSPA5-MYC) fusion with MYC. $\mathbf{c}$ HeLa cells were transfected with the indicated constructs and treated with MG132 for $12 \mathrm{~h}$. Total cell lysates were harvested for IP and western blot analysis with the indicated antibodies. d HeLa cells were transfected with the indicated constructs and then subjected to an in vivo ubiquitination assay. e Western blot analysis of GP78 and HSPA5 expression (top) and the measurement of the migration and invasion abilities by transwell assays (bottom) in MCF7 cells with overexpressing HSPA5, HSPA5/GP78 or HSPA5/GP78R2M, respectively. Data are shown as mean \pm s.d. of three independent experiments performed in triplicate. $* * P=0.01 ; * * * P=0.001$

Sup. 2b

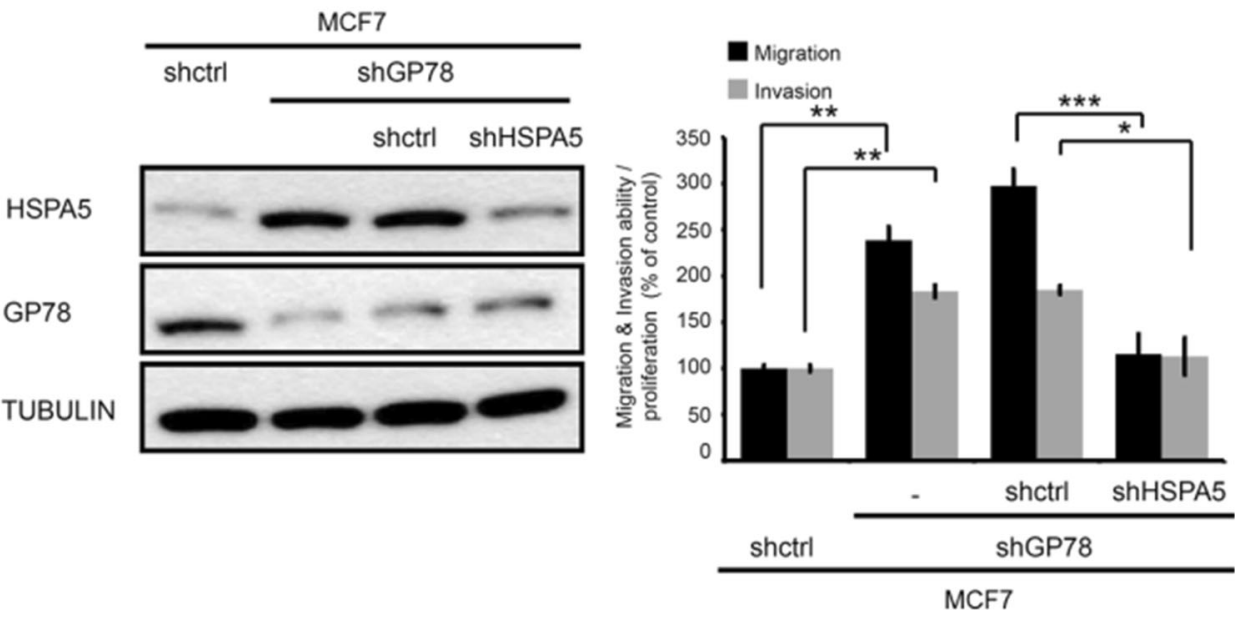

